\title{
Les pratiques langagières des enseignants : des savoirs professionnels inédits en formation
}

\author{
D. Bucheton, A. Bronner, D. Broussal, A. Jorro, M. Larguier \\ LIRDEF- ERT, IUFM de Montpellier ${ }^{1}$
}

Résumé : Dans les IUFM nous formons de futurs professionnels de l'enseignement, métier dans lequel la parole est un instrument de travail essentiel, le médiateur principal des interactions maître/élèves lors des apprentissages des élèves. Savons nous préparer les enseignants à ces conduites langagières de la classe? Savons-nous repérer et analyser l'impact des gestes langagiers et corporels dans la construction des apprentissages ? L'article fait état d'une recherche en cours sur cette question. Il propose une théorisation de la parole de l'enseignant dans la classe et montre quelques problèmes qu'elle soulève chez les stagiaires dans le moment particulier et précisément étudié des débuts de cours. Dans le cadre de cet article, il s'agit d'un début de cours en mathématiques.

Mot-clé : geste langagier, interaction maître-élève, corporéité de l'activité enseignante.

\section{Des postulats et discours théoriques partagés à la réalité de la formation et à ses effets.}

\subsection{Une certaine communauté de pratique en formation : Le rôle structurant du langage}

Une certaine communauté de vue et de pratiques se dégage de colloques récents pour souligner le rôle des langages divers comme vecteurs du développement cognitif, identitaire, et par effet retour vecteur du développement du langage.

Le colloque de Perpignan 1998 : Parler écrire pour penser, apprendre et se construire: l'oral et l'écrit réflexifs (Chabanne et Bucheton 2002), a montré l'importance des pratiques langagières réflexives pour le développement des apprentissages corrélés au développement général du sujet. Celui de Bordeaux (Jaubert, Rebière et Bernié 2003), a mis en travail la notion de communauté discursive soulignant le lien constitutif entre le langage, ce qu'il exprime et l'espace épistémologique et social dans lequel il s'inscrit. Il a contribué à problématiser le lien étroit qui unit les pratiques langagières orales et écrites aux communautés de pratiques (Lave 1991, Lave et Wenger 1991), épistémologiquement et culturellement instituées. Ces deux colloques ont montré comment l'apprentissage se joue

\footnotetext{
${ }^{1}$ L'article fait état d'une recherche en cours faite dans le cadre d'une Equipe de Recherche Technologique. Le thème en est l'étude des débuts de cours, conditions d'entrée dans les apprentissages. L'équipe est pluridisciplinaire et rassemble des chercheurs de l'IUFM de Bordeaux, Montpellier, Aix-marseille.
} 
dans la participation du sujet comme acteur dans des pratiques culturelles dont il s'approprie les modes de dire, de penser et de faire. Ils ont montré le rôle décisif du langage pour s'inscrire dans ces communautés de pratiques qui, dans les disciplines enseignées à l'école (y compris les disciplines artistiques ou sportives), sont en même temps des communautés discursives.

On peut ainsi inventorier chez les formateurs IUFM, des pratiques langagières (mémoire professionnel, diverses formes d'analyse de pratiques orales ou écrites, qui mettent en travail ce rôle structurant du langage pour objectiver et formaliser des savoirs professionnels. Elles sont construites selon les trois mêmes principes de base hérités du socio-constructivisme vygotskien et bakhtinien :

1. La parole singulière de l'apprenant (orale et écrite) est un vecteur central de son entrée dans les apprentissages et de son développement cognitif, langagier, psycho-socio affectif et identitaire,

2. Cette parole singulière travaille avec, dans, contre une communauté de pratiques qui est aussi une communauté discursive. Elle s'institue et se construit dans des cadres spécifiques qu'elle modifie et réélabore en permanence (Goffman 1974).

3. Elle a besoin d'être étayée pour se développer. En d'autres termes la présence d'un formateur lui même porteur d'une culture est nécessaire pour que l'expérience du sujet se transforme.

\subsection{Mais des constats inquiétants}

\subsubsection{TB à l'écrit, TB à l'oral, en pratique : peut beaucoup mieux faire}

Nous avons largement mis en œuvre ces dispositifs dans la discipline du français, engageant le travail réflexif de l'écrit : mémoire professionnel, portfolio, journal de bord. Nous avons pu montrer qu'ils développaient des postures réflexives et professionnelles intéressantes, qu'ils permettaient de conceptualiser, nommer des savoirs didactiques et notionnels (Bucheton 2001-2003). Pour autant on ne peut affirmer la nature de l'impact qu'ils ont sur le travail langagier propre de l'enseignant dans la conduite de la classe et les problèmes qu'il a à résoudre.

Ainsi, des stagiaires peuvent avoir fait d'excellents mémoires, d'excellents journaux de bord, avoir une aisance verbale étonnante dans les entretiens, les groupes d'analyse de pratiques, les exposés ou communications diverses, et se révéler très « démunis » devant la classe alors même que des stagiaires silencieux ou discrets peuvent s'avérer «performants » (termes utilisés dans leur sens usuel). Ceux-là ont appris autrement ou ont appris d'autres choses.

Les effets formateurs pour la conduite langagière de la classe de ces dispositifs oraux ou écrits ne sont pas démontrés. Chaliès (2002) montre par exemple que les entretiens avec le tuteur apprennent aux stagiaires à "parler avec le tuteur et à comprendre ses stratégies ! », ils confortent les savoirs compris, acquis par la pratique. Dans les journaux de bord tenus au quotidien pendant le stage de 3 semaines en responsabilité, on constate en effet une certaine ouverture et diversification des postures d'enseignement. Rien ne nous dit si c'est la pratique ou la dimension réflexive ou les deux qui produisent ces déplacements.

1.2.1. Les savoirs professionnels institués en formation sont très fragiles. La visite de classes de titulaires lors de leurs premières années d'enseignement montre par exemple que l'écriture au quotidien, dans toutes les disciplines, telle qu'elle est préconisée en formation ou dans les I.O. est très peu mise en place. 

inégale.

1.2.2. La transformation de l'expérience de la classe en savoirs professionnels est L'étude des journaux de bord ${ }^{2}$ tenus pendant les trois semaines d'un stage de formation en responsabilité (Bucheton, Chabanne 2002) a pu montrer combien était faible la mobilisation dans la pratique des savoirs didactiques, enseignés pendant les cours «théoriques » de première ou deuxième année ; combien était difficile, lent et très inégal d'un stagiaire à l'autre, ce passage entre l'action dans la classe et la forte implication qu'il demande et la transformation de cette expérience en conceptions pragmatiques: l'émergence de savoirs professionnels, didactiques, décontextualisables et donc mobilisables et questionnables.

\subsection{Une double hypothèse :}

\subsubsection{La diversité des pratiques et discours de formation devient un frein.}

Les pratiques de formation, les savoirs mobilisés, les pratiques professionnelles montrées par les divers partenaires de la formation sont éclatée, chaotiques, voire conflictuels. Si l'institution développe un discours théorique de façade commun, il n'y a pas de communauté de savoirs, ni de pratiques, encore moins de communauté discursive entre les formateurs. Cette diversité loin de constituer une richesse se révèle être un frein .

Trois logiques sont mises en œuvre ouvrant trois niveaux de conceptualisation de l'action dans la classe. Une logique modélisante dans les divers modules didactiques (leçon modèle, idéale), une logique réflexive : analytique, généraliste et décontextualisée dans les analyses de pratiques d'obédiences diverses avec ou sans vidéo (Tochon 2002), une logique de compagnonnage portée par les tuteurs de stage, faite souvent de monstration en contexte de micro-gestes et techniques propres à la classe. Ces trois logiques, renvoient à trois systèmes de savoirs (notion d'écosystème de connaissances : Lemke, 1997), soutenus par des types de discours et des pratiques différentes. Contrairement à ce qui est postulé par le système mis en place en IUFM, ces logiques ne se complémentent pas spontanément. L'alternance n'est pas intégrative. Elles se superposent et n'entrent pas en réseau, voire peuvent s'annuler ou devenir conflictuelles. «Mettez-vous d'accord», disent les stagiaires qui reçoivent parfois en une semaine des rapports de visite de stage diamétralement opposés.

\subsubsection{Former à la conduite de la classe est un objet de savoir encore très flou.}

Les modèles descriptifs des caractéristiques des gestes du métier pour faire apprendre les élèves, servant de référence pour la formation ont un caractère très générique et hautement discutable. Ils ne relèvent pas d'analyses ergonomiques du métier d'enseigner tel que celui-ci est pratiqué (quelque chose comme des genres professionnels (Clot 1995) identifiables et largement partagés par toute la communauté et pouvant être objets de discussion et de formation). Autrement dit, les savoirs professionnels que nous sommes censés faire acquérir ne sont qu'assez peu identifiés, encore moins partagés sauf en termes très génériques et vagues de compétences. Le référentiel français ${ }^{3}$ en repère près d'une quarantaine, regroupés

\footnotetext{
${ }^{2}$ Pendant tout le stage en responsabilité les stagiaires ont tenu au quotidien, sans prescription de forme un journal de stage faisant état de leurs pratiques, remarques, ajustements dans le domaine de la didactique du français

${ }^{3}$ Ministère de l'Education nationale (1994), Annexe III du BO n 43 du 24 novembre 1994, « Référentiel des compétences professionnelles du professeur des écoles stagiaire en fin de formation initiale ».
} 
en 4 catégories, le Ministère de l'Education Québécois douze ${ }^{4}$, Perrenoud en dénombre une dizaine $^{5}$. Ces compétences décontextualisées renvoient à des postures pédagogiques, éthiques, transversales mais non aux gestes didactiques spécifiques des disciplines ( item 25 : se mettre à l'écoute des élèves et développer une écoute mutuelle dans la classe). Elles sont peu opératoires pour préparer un cours, gérer l'hétérogénéité de la classe, s'ajuster aux élèves, analyser leurs échecs ( item 10: identifier les obstacles que peuvent rencontrer les élèves, ceux notamment liés aux représentations et à une maîtrise insuffisante de la langue). Prenons l'exemple du débat tel que les nouvelles I.O. du primaire français le préconisent. Il nécessite des formes d'adresse, de questionnement, de focalisation, de guidage très spécifique selon son contenu littéraire, scientifique, mathématique, historique, voire philosophique. Il s'agit dans tous les cas de donner la parole aux élèves mais pour leur faire adopter des postures interprétatives, argumentatives, des modes de réfléchir et d'échanger spécifiques des disciplines et des cycles d'enseignement concernés. En d'autres termes, on forme à des principes non à des pratiques dont on ignore le degré de réalité dans les établissements et les équipes pédagogiques.

Nous partageons ici le point de vue de Clot (1998) selon lesquels d'une part il n'est pas possible d'accompagner une formation professionnelle sans une connaissance approfondie du travail, d'autre part, il convient de distinguer ce qui peut être abordable en formation initiale de ce qui peut être travaillé en formation continue où on peut s'appuyer sur un noyau de procédures expertes déjà là (Pastré $2004^{6}$ ).

Ajoutons à cela que le passage des savoirs requis pour passer les concours aux savoirs professionnels construits en deuxième année est loin d'être naturel.

En première année la préparation à un concours, requiert des savoirs théoriques relevant des disciplines et des savoirs relatifs à la façon d'enseigner : les modèles didactiques abstraits, décontextualisés, de la pratique de la classe. Cette culture disciplinaire de l'enseignant, est supposée constituer des savoirs fondateurs d'arrière plan auxquels devraient s'arrimer pour se structurer en deuxième année les savoirs professionnels d'ordre plus technique, nécessaires à la conduite de la classe.

C'est sur ce deuxième objet de savoir, spécifique de la formation professionnelle de $2^{\text {ème }}$ année que nous voudrions faire porter notre réflexion ici.

Ce travail précis, ergonomique et didactiques de description à grain d'analyse très fin des conduites de classe réelles, dans des situations identifiées a commencé. Il s'agit selon nous de descendre jusqu'aux modes d'agir et de décider des enseignants dans des types de situation ou de contextes plus précisément identifiés. C'est ainsi que des didacticiens des mathématiques lancent l'idée d'un observatoire des pratiques réelles. Ce travail d'analyse s'inscrit dans une longue tradition de recherches sur les fonctions de la parole de l'enseignant, (Postic, Altet, Nonnon etc. Il devrait permettre d'envisager d'autres contenus et modalités de formation, d'autres pratiques langagières pour y parvenir. C'est dans cette direction que travaille notre ERT (équipe de recherche technologique).

\subsection{D'où la proposition : changer de logique de formation}

1.4.1. Hypothèse 1: le langage est la dimension centrale de l'activité de l'enseignant. Les problèmes des stagiaires se manifestant dans et au travers des conduites verbales de la classe - l'action du maître est fondamentalement langagière y compris quand il

\footnotetext{
${ }^{4}$ Ministère de l'Education du Québec (2001), La formation à l'enseignement, les orientations, les compétences professionnelles.

${ }^{5}$ PERRENOUD P. (1999), Dix nouvelles compétences pour enseigner, ESF.

${ }^{6}$ PASTRE, P.( Juin 2004) Communication lors du séminaire du LIRDEF à Montpellier.
} 
se tait - cette médiation langagière et toutes les significations qu'elle véhicule, construit ou rend possible, doit devenir l'objet fédérateur de la formation.

1.4.2. hypothèse 2 : Travailler des situations problématiques : un projet de formation commun pour les stagiaires et les formateurs. Ces situations, emblématiques et charnières, travaillées avec les différents acteurs de la formation devraient permettre la construction partagée et la mise en réseau de savoirs d'ordre divers. Une logique de formation intégrative (Aghulon 2002) pourrait ainsi se mettre en place.

D'une logique cumulative des savoirs, impossible à gérer en un laps de temps de formation aussi réduit, on propose de passer à un ciblage des difficultés les plus communément rencontrées par les stagiaires dans la conduite de la classe. Cette conception nécessite d'identifier des classes de problèmes, des classes de situations les plus à risques, didactiques ou non didactiques. L'entrée dans l'écrit en fait partie, débuter ou clore une leçon aussi, enseigner en ZEP également. Etc.

L'oral et l'écrit en formation ont alors pour finalité, à l'occasion du travail sur ces situations spécifiques, de tisser le lien entre pratique située et réflexivité hors contexte.

\section{Le cadre de la recherche en cours : perspectives, méthodologie}

L'étude poursuivie dans le cadre de l'ERT porte sur les conduites de classe langagières et non langagières des stagiaires dans une situation de formation spécifique : les débuts de cours pendant le stage pratique en responsabilité. Nous postulons que l'analyse de ces conduites langagières (les brouillonnments oraux et écrits, les ajustements divers aux réactions et réponses des élèves) permet d'ouvrir une fenêtre sur les conceptions en actes (concepts pragmatiques: Pastré 2002, les représentations des problèmes didactiques, relationnels, spatio-temporels etc ; ces problèmes devant être résolus pour mener à bien les apprentissages précis visés par la leçon. L'autoconfrontation (conduite par un formateur) ou l'hétéroconfrontation (avec des pairs dans un GAP) permet le retour réflexif sur l'activité intra-psychique (observations, émotion, dilemmes, prises de décision, négociation de sa propre «face » développée pendant l'action.)

Ces situations observées sont en effet problématiques à plus d'un titre :

- Elles ne font l'objet d'aucune préparation. Elles sont impensées.

- C'est le moment d'installation de divers « instruments pour la classe ».

- C'est un moment de trac, de fragilité émotionnelle y compris pour l'expert

- Le début de cours est un triple seuil, (Brenas 2003) un passage souvent en trois phases entre le dehors de la classe et les règles du dedans (une installation corporelle et matérielle) l'entrée dans un cadre épistémique (on est là pour apprendre : le travail commence), l'entrée dans un cadre épistémologique spécifique ( une lecture littéraire et non historique par exemple. Le passage de ces différents seuils par les élèves est loin d'être synchrone.

- Selon les cadres didactiques des disciplines, le type de mise en activité des élèves diffère. La phase souvent dite de problématisation recouvre des conceptions fort différentes, nécessite des gestes d'étude des élèves spécifiques.

L'objet de la recherche en cours est multiple: identifier des savoir-faire professionnels langagiers fondateurs dans un moment de difficulté ; repérer les réussites, les obstacles, les stratégies des stagiaires; faire de l'analyse de ce moment précis de la classe un objet de formation. L'objet de la recherche est technologique en ce sens que nous devons construire des instruments de formation devant répondre à un problème de formation précisément identifié. 
Par ce biais nous travaillons deux types de réflexivité langagière. L'une est située, dans l'action didactique, elle est constitutive de la dynamique de l'action, des dilemmes et prises de décision rapides lorsque surgissent des imprévus. L'autre est seconde, située, elle aussi, mais dans un tout autre contexte (Broussal 2004), soumise à une autre dynamique. Elle peut permettre plus de distanciation mais n'évacue pas l'émotion du langage en action. C'est ce processus de secondarisation (Jaubert et Rebière 2002 ) qui est véritablement formateur. Les cercles d'étude vidéo deviennent alors un moment réflexif, discursif sur le discours de et dans l'action. Le langage de l'enseignant pour la conduite de la classe devient alors un contenu de formation enseigné.

Nous en proposons maintenant une théorisation provisoire. Nous développerons plus succinctement les unités d'analyse que nous avons retenues pour donner quelques exemples de nos premiers résultats.

\section{1. La parole de l'enseignant comme médiation fondamentale pour le développement et les apprentissages des élèves}

1) Ce truisme ne va pas de soi. Les enseignements de type Coopératif (pédagogie institutionnelle) le remettent par exemple fortement en cause.

2) Ce postulat ne signifie pas que la parole du maître soit dominante (et assommante !). Voici sept propositions pour définir cette médiation de la parole enseignante

2..1.1. La parole de l'enseignant est une médiation fondatrice. La construction des savoirs scolaires est tributaire de la mise en place d'un dialogue pédagogique responsif (Bakhtine1979). Le discours de l'enseignant mobilise tel ou tel autre rapport aux objets d'étude (Boiron 2004), installe les élèves dans telle ou telle autre posture pratique, ludique, créative, réflexive (d'exploration, problématisation, conceptualisation, etc) (Bucheton 2001 )

Ce dialogue est fondamentalement asymétrique. Le rôle d'étayage de la parole de l' adulte est central (Vygotski). Cet étayage se construit par la mise en place de scénarios $\left(\right.$ Bruner $\left.^{7}\right)$ de paroles, gestes, actions partagées (ex «passe au tableau »).,

Ce dialogue est complexe au sens ou ses composantes langagières, cognitives, socioaffectives, institutionnelles (les jeux de place et rôles) sont étroitement corrélés. Nous avons montré dans le cadre de la didactique de l'écriture comment les développements langagiers, psycho-affectifs, identitaires, cognitifs étaient fortement corrélés ( Bucheton 1995).

2.1.2. Cette médiation est une action située. Sa caractéristique principale est donc le principe d'ajustement pragmatique.

Elle porte la marque du lieu spécifique, mais aussi de l'instant, (d'où l'étude de moments de classe spécifiques). Elle s'inscrit dans une durée, une expérience partagée des sujets mais aussi de l'institution et de ses évolutions ( l'enseignement de la lecture à l'école primaire porte la marque des nouveaux programmes de littérature).

Elle contient et se construit dans des contenus didactiques précis et spécifiques des disciplines enseignés. D'où pour l'étudier la nécessité d'une analyse didactique a-priori .

Elle s'ajuste aux modes de dire et de faire des élèves, aux types de regroupement d'élèves, types d'école, aux scènes scolaires diverses sur lesquelles elle s'exerce. Dans une même situation de classe l'enseignant peut circuler sur la scène frontale (toute la classe), sur la scène duale (dialogue spécifique avec un ou deux élèves à une table), dans les scènes de

\footnotetext{
${ }^{7}$ Qui renvoient à l'idée d' ethno-méthodes culturellement mises en place.
} 
groupes restreints, il peut aussi surveiller de près et intervenir sur les scènes de coulisse où se jouent entre les élèves des jeux divers, tout en observant la scène privée (qui se manifeste par les regards et le corps) des élèves silencieux .

De ce fait la parole de l'enseignant met en jeu des jeux (Hannoun 1989), registres, tonalités de langage multiples. Elle est adaptative et constamment inventive.

2.1.3. Elle est socialement et culturellement construite dans l'histoire croisée des acteurs (maîtres-élèves, acteurs divers du système) et de l'institution. Cette culture de l'école s'inscrit dans une architecture complexe de divers cadres emboîtés (Goffman 1974), en partie objectivables et contractualisables, en partie peu formalisables et verbalisables car enfouis dans l'histoire sociale et scolaire des sujets : cadres politiques, institutionnels, socioscolaires, didactiques. Ces cadres sont perçus, construits, mais aussi agis. Autrement dit, ils sont modifiables et évolutifs par le jeu même de la dynamique dialogique mise en place. La médiation du dialogue responsif de l'enseignant se construit dans ces doxas, habitus, institutions diverses qu'elle véhicule et transmet de manière largement inconsciente d'où un certain nombre de résistances qui ne relèvent pas d'une intentionnalité affirmée.

2.1.4. Cette médiation de la parole est épaisse : c'est un agir pratique, communicationnel, relationnel, réflexif. En d'autres termes les actes de langage de l'enseignant largement étudiés ou discutés depuis plus de vingt ans ont cette spécificité d'être porteurs de significations multiples et enchassées, ouvrant toutes sortes de modes de signifier (François 2004). D'où la notion de pluri-agenda (plusieurs choses à faire dans le même acte de langage : évaluer, réconforter, orienter, formuler une question nouvelle, étayer et ponctuer, s'adresser à un élève et à l'ensemble de la classe). Dans la même unité didactique de quelques minutes, l'enseignant doit gérer les tâches et les contrats didactiques qui les sous-tendent, l'évaluation de l'avancée du travail, les faces et relations des élèves, la ponctuation et le tissage entre les diverses unités de la leçon. Cette épaisseur de la parole du maître est lente à se mettre en place. Elle est pour le jeune enseignant le lieu de dilemmes non résolus. On peut faire l'hypothèse que si les jeunes enseignants parlent trop en classe c'est en partie parce qu'ils ont du mal à gérer cette polysémie constitutive de la parole au travail.

2.1.5. Elle est hétéronome. La caractéristique de la parole dans la classe est cette hétéronomie sémiotique (Bucheton 2002 HDR) : elle mêle divers vecteurs de cette médiation, en particulier le corps parlant de l'enseignant (Jorro 2004), des langages graphiques multiples, des instruments didactiques verbaux et non verbaux. En maternelle et en cycle 2, on peut penser que cette hétéronomie est décisive.

\subsubsection{Elle est dynamique.}

Si le déroulement du «texte $d u \operatorname{cours}^{8} »$, sa mise en scène en diverses unités didactiques, inscrites dans le temps précis de la leçon peut être planifié dans la préparation, il ne peut pas être pré-écrit. Etant co-élaboré dans l'action langagière et non langagière avec les élèves, sa dynamique propre est imprévisible et est le fruit même des interactions, ajustements, négociations du sens qui s'y jouent et s'y construisent. Les dilemmes que l'enseignant gère dans cette dynamique (ex: j'interromps le cours et je réexplique la consigne), ont leur pendant chez les élèves qui en vivent d'autres (ex : j'écoute les explications ou la correction ou je finis la liste d'exercices qu'on vient de me donner). Les gestes didactiques du maître pour mettre en place un geste d'étude chez les élèves (ex : tenter de faire émerger un questionnement sur un texte historique ou littéraire), ne provoquent pas forcément la

\footnotetext{
${ }^{8}$ Dans la mesure où le cours est supporté par un ensemble de paroles, ayant une ouverture et une clôture, nous le considérons comme « un texte ».
} 
dynamique attendue. Les élèves par leur mode d'implication, leurs postures scolaires déjà là, leurs résistances diverses, modifient, voire détournent les finalités initiales de la leçon ou des exercices proposés. Ils se donnent d'autres tâches, le maître corrige et modifie les siennes. Une large part du texte de la classe est imprévisible notamment pour le novice. Cette dynamique interactionnelle est en partie cognitive, en partie socio-affective et relationnelle, en partie physiologique (fatigue, rythme). Des jeux complexes d'engagement, désengagement ponctuel ou durable des acteurs (maître et élèves) la structurent. Apprendre c'est entrer dans des jeux de sémiotisation que supporte en grande partie le langage et ses entours .

\subsection{7..La dynamique langagière, actionnelle et cognitive se joue dans les écarts, les} failles, la bizarrerie, dans l'ouverture de possibles nouveaux du sens ou de l'action. Ce dialogisme asymétrique fondamental de la médiation didactique trouve sa puissance maximale dans des ajustements négociés sur des zones de faille, de déstabilisation, de savoirs non partagés ou questionnables. D'où la nécessité pour le maître d'être en mesure de repérer ces lieux d'intervention didactique à privilégier. Ils se manifestent dans divers «événements ${ }^{9} »$. Le texte de la classe, inscrit dans un temps, dans un espace spécifique, joué par des acteurs divers, se déroule sous la forme d'un récit (il est d'ailleurs racontable a posteriori). Ce récit est ponctué d'événements partagés (didactiques ou non) qui le réoriente et au travers desquels les significations se construisent à la fois collectivement et de manière singulière pour chaque acteur. Ce sont probablement les lieux principaux où se construisent pour l'élève le sens des objets travaillés, le sens de l'école, le sens de la place qu'on y tient, le sens de la relation didactique. Ce sont eux qui provoquent les mouvements discursifs créant la dynamique cognitive et langagière. D'où notre hypothèse d'en faire un objet pivot d'étude et de formation.

\section{Conclusions intermédiaires}

1. Cet apprentissage de la parole de l'enseignant ainsi conçue, instrument médiateur de la mise en activité des élèves n'est pas encore un objet d'étude commun pour les formateurs. Tout au plus quelques dimensions techniques ou décontextualisées sont travaillées (par exemple les modules « la voix, le corps » à l'IUFM de Montpellier).

2. Nous faisons l'hypothèse qu'une part importante de la professionnalisation du métier d'enseignant se joue dans ces gestes d'ajustement, d'écoute, de négociation patiente du sens des tâches, des situations, des savoirs avec les élèves dans leur différence. Savoir les écouter pour savoir leur parler et étayer au mieux leurs apprentissages est de première importance. L'enseignant expert est celui qui sait s'ajuster tout en restant tenace sur les enjeux didactiques à construire.

3. Il reste à trouver en formation initiale les situations, les formes d'étayage qui permettent le développement de cette composante de la professionnalité. La video-scopie, l'arrêt et le retour sur image permettent cette descente dans le grain d'analyse nécessaire pour comprendre l'architecture complexe des cadres multiples déclarés ou non qui structurent les prises de décision, et les ajustements dans l'action. Mais on ne sait pas encore quel peut être le degré de perception, de compréhension, d'interprétation de ces gestes d'ajustement en formation initiale. Confrontés à la même vidéo d'une situation d'enseignement experts et novices n'en tirent pas les mêmes informations, les mêmes questions. Les savoirs d'expérience des uns ou les modèles théoriques des autres sont autant de filtres opacifiants, nécessaires, et à remettre en question.

\footnotetext{
${ }^{9}$ Nous préférons le concept d'événement à celui d'incident critique d'où il est en partie tiré. L'incident c'est l'accident dont il faut se prémunir. L'événement c'est la vie, la norme de ce qui provoque la dynamique du récit ou de l'interaction. Il ne s'agit pas en classe d'entendre ronronner le moteur.
} 
4. Un problème : l'œil des novices, insuffisamment frotté à l'expérience, à la perception du vécu de la classe ne peut pas encore distinguer certains détails pourtant essentiels pour l'expert. Sa représentation pragmatique est naissante. Comment alors lui procurer cette expérience en accéléré pour aiguiser sa perception ? Quelles simulations sont nécessaires pour l'entraîner à réagir aux événements toujours complexes qui surviennent?

5. Ces gestes d'ajustement qui constituent le fondement de ce dialogue responsif de l'adulte sont aussi le lieu où se fédèrent et s'articulent la diversité des enseignements reçus en formation initiale (psychologiques, didactiques, institutionnels, sociologiques, épistémologiques etc). La non articulation de ces savoirs donne la mesure des effets du système de formation.

\section{Un début de cours problématique}

\subsection{L'analyse de la situation mathématique Une analyse d'A. Bronner et Myrène Larguier}

$1^{\circ}$ ) La rencontre avec le savoir, médiatisée par le langagier :

La séance dont nous analysons un extrait, est une séance de mathématiques en classe de CE1, menée par une stagiaire en $2^{\text {ème }}$ année d'IUFM. Cette séance est une adaptation et une mise en scène d'une situation décrite dans ERMEL (2001). Elle est analysée selon le point de vue et les outils de l'approche anthropologique (Chevallard 1992, 1999). Cette approche permet de décrire la séance en unités qui correspondent aux différentes tâches dans lesquelles sont engagés élèves et maître et d'analyser l'organisation mathématique, réponse à la question : «quels sont les apprentissages visés par la séance?». D'autre part, elle permet aussi d'analyser l'organisation didactique et en conséquence les gestes professionnels de l'enseignante en réponse à la question : «comment enseigner telle notion ? ». Les concepts de temps didactique, de topogénèse et de chronogénèse (Chevallard, 1985) sont utiles pour penser les rôles respectifs des élèves et du maître, en ce qui concerne leur place réelle, et la façon dont ils prennent part à l'avancée du temps didactique.

Nous nous appuierons aussi sur la théorie des situations didactiques (Brousseau 1986), notamment les notions théoriques de contrat didactique, de milieu et de dévolution seront convoquées pour analyser la façon dont l'enseignante met en scène la situation en interaction avec les élèves, à travers la médiation langagière.

L'épisode évoqué dans cet article est celui de la constitution de la communauté discursive dans les premiers instants de la séance. Il se situe dans ce que l'on peut qualifier de «mise en place du milieu de la situation ». Cet épisode se déroule dans les deux premières minutes. La tâche est donnée oralement par l'enseignante, c'est le rappel d'un travail réalisé la veille :

ENSEIGNANTE : on a regardé ++ c(e) qu'i(l) y avait sur cette carte...[...] celle que vous avez +++ alors qui peut me rappeler ce qu'on voit sur cette carte.

ENSEIGNANTE : Camille ::

CAMILLE : ben + on voit des <traits?> + et on voit des lignes <.... ?> +++ et puis aussi y'a :: + y'a des numéros +++ et puis on voit plusieurs pays

L'enseignante semble chercher à construire un milieu en réactivant la mémoire didactique de la classe, en lien avec l'activité de la veille avec l'objectif d'aboutir à remettre les élèves dans le même contexte. Elle attend que les élèves, par ce jeu d'articulation avec la séance précédente, s'inscrivent dans le contrat didactique (Brousseau 1986) : qu'on pourrait formuler ainsi «on refait ce qui a été fait hier, on doit traduire les nombres indiqués entre deux noms sur le schéma comme la distance en km entre deux villes».

Mais le milieu n'est pas assez bien construit et permet aux élèves d'autres interprétations sur la tâche, en adéquation avec la consigne réellement formulée. En effet les verbes « regarder » et « voir » détournent les élèves vers une tâche d'énumération de ce tout ce qui est observable. 
La tâche que l'enseignante veut réinitialiser n'est donc pas la tâche comprise par les élèves, et l'enseignante est alors dans l'obligation de faire avancer le temps didactique, ce qu'elle fait en privilégiant les réponses d'élèves qui confortent son projet et en ignorant des erreurs d'interprétation qui le ralentiraient. Il s'agit ici, à travers les échanges verbaux et les interactions avec le milieu, d'un geste professionnel, peut-être pas encore conscientisé.

ENSEIGNANTE : on voit des numéros et qu'est-ce qu'on voit je t'ai pas entendue

CAMILLE : plusieurs pays

ENSEIGNANTE : plusieurs pays ++ alors

UN ELEVE : non

UN ELEVE : non

UN ELEVE : c'est des villes

ENSEIGNANTE : est-ce que c'est des, pays

UN ELEVE : des villes

UN ELEVE : non

ENSEIGNANTE : des villes ++ c'est les villes + oui + c'est des villes + et les numéros c'est quoi

CAMILLE : c'est + les 〈co ?>

ENSEIGNANTE : les chiffres que tu vois

CAMILLE: ben euh ::

UN ELEVE : c'est

ENSEIGNANTE : CHUT :::

CAMILLE : c'est les kilomètres qu'il faut faire

ENSEIGNANTE : c'est le nombre de kilomètres oui

YVON : la distance de ::

ENSEIGNANTE : c'est la distance + tout à fait Yvon +++ c'est la distance ++++ c'est la distance qu'il y a

L'enseignante profite de l'élève qui affirme que ce sont des villes pour reprendre cette interprétation, et amener le terme «juste » dans le milieu, et à travers ce terme, le concept adapté à la dimension épistémologique de la situation. On voit ici le travail langagier, conscient ou inconscient, guidé par les intentions didactiques de l'enseignante, l'amenant à « forcer » un premier changement de cadres : de celui des pays ou d'une géographie flottante à celui d'un pays avec ses villes selon une modélisation sous-jacente à la carte.

.L'enseignante enchaîne sur les «numéros » en restant d'ailleurs dans le vocabulaire de l'élève sans chercher à faire rectifier le terme «numéro » qui est inexact. Elle prend peut-être conscience de ce manque de rigueur dans son vocabulaire et impose un premier glissement de sens, permis par le travail langagier, à l'insu des élèves, à travers les dénominations «chiffre », et enfin «nombre de kilomètres ». On repère là un phénomène qui se reproduira plusieurs fois dans la séance, c'est le manque d'anticipation sur le vocabulaire à mettre en place. En effet, le milieu objectif pour l'élève comprend les représentations sémiotiques (Duval 1993) liées à la carte de géographie, mais aussi ce qui permet l'accès aux savoirs visés, autrement dit les termes et le langage mathématique adéquats. Ces éléments, constituants essentiels du milieu, auraient pu être prévus dans le discours des élèves et de l'enseignante elle-même avant la séance. Ici le vocabulaire spécifique (nombre de $\mathrm{km}$, distance d'une ville à une autre, longueur, etc...) aurait pu être repéré dans un travail préparatoire. Le milieu aurait été mieux contextualisé grâce à ces savoirs.

Le rôle d'Yvon est déterminant pour déclencher le passage à la deuxième phase dans laquelle une nouvelle tâche va être donnée aux élèves et règle ainsi l'incertitude mathématique et langagière de la situation. Yvon a le mot juste «la distance de » et devient le passeur pour le cadre des distances, cet autre monde dans lequel doit se mouvoir la situation avec ses acteurs pour atteindre les objets de savoir. L'enseignante a enfin l'élément de liaison espéré entre la séance de la veille et ce nouveau cadre. Le sauveur est remercié implicitement pour son rôle décisif dans la chronogénèse de la séance " c'est la distance + tout à fait Yvon +++ c'est la distance ++++ c'est la distance qu'il y a ». La séance pourra alors reprendre son cours et l'enseignante s'autorise à poser la question qui initialise une deuxième phase qui concerne le 
véritable enjeu de la séance à savoir : « voilà ++ et si je vous demande +++ quelle / est / la / distance + entre Paris + et Lille ».

Dans la première phase on peut faire l'hypothèse que l'enseignante veut une collaboration avec des élèves pour assurer la dévolution de la situation, même s'il se construit un leurre puisque enseignante et élèves n'interprètent pas la même tâche. L'élève a la responsabilité de deviner les attentes du professeur, à ce jeu le gagnant du moment est Yvon qui a su «voir » exactement ce qui devait être vu selon le professeur. Si une bonne dévolution (Brousseau 1986) est un processus qui permet de limiter au maximum les interprétations de l'élève au sujet des attentes du professeur, on peut dire que dans cette première phase, le processus de dévolution n'est pas un geste professionnel facile à réaliser pour un professeur débutant.

En terme de formation initiale, l'analyse de cet épisode à partir de la vidéo, ou à partir du protocole écrit, et notamment des interactions langagières, peut amener à faire prendre conscience, d'une part du décalage éventuel qui peut exister entre la tâche donnée par l'enseignant et la tâche dans laquelle les élèves s'engagent, et d'autre part des problèmes qui se posent pour réussir une bonne dévolution. Un geste professionnel peut alors être dégagé, celui de réaliser une analyse a priori d'une séance et de savoir en particulier anticiper, non seulement sur l'agir mais aussi sur le dire. En particulier anticiper :

- la formulation précise des consignes et le moyen de les communiquer aux élèves ;

- les erreurs de compréhension des tâches à cause d'un milieu de la situation dont les frontières restent floues ;

- le vocabulaire spécifique objet d'apprentissage pour les élèves.

Un autre geste professionnel peut aussi être mis à jour pour être interrogé dans la partie du scénario de début de cours: construire et conduire l'articulation de deux séances de disciplines différentes et donc supportant des apprentissages relevant d'épistémologies différentes

L'approche anthropologique nous amène à faire l'hypothèse que le travail réflexif à partir des pratiques langagières des séances analysées a posteriori en formation initiale, permet d'identifier des gestes professionnels comme étant des savoir-faire ou des techniques dans les tâches d'enseignement, gestes repérés eux-mêmes comme des tâches d'enseignement dont il faut essayer de mettre à jour les éléments théoriques qui les fondent.

\subsection{La corporéité de l'activité enseignante : une analyse d'Anne Jorro...}

Dans cette seconde analyse, l'orientation du questionnement ne réside plus tant dans une épistémologie des savoirs - ce que l'analyse didactique a mis en évidence - mais plutôt dans une épistémologie du pouvoir d'agir de l'enseignant, là où les savoirs d'action caractérisent le professionnel (Schön, 1996). En effet, le professeur investit la situation avec une rationalité limitée, il s'engage non pas sur le seul plan conceptuel mais aussi sur les plans psychologique, affectif, social, cherchant à mobiliser les élèves, à organiser la dévolution. Le pluri- agenda de l'enseignant est en jeu au point que l'enseignant n'apparaît plus comme un regard et une voix destinés à la classe mais expose sa corporéité, un corps parlant et regardant qui affecte la perception des élèves.

La corporéité de l'activité enseignante reste encore peu interrogée par la recherche, les travaux de Pujade -Rernaud, (1981) avaient mis en évidence la place du corps de l'enseignant en classe ainsi que son incidence du point de vue de la communication ceux de Luciano-bret (1991), sa dimension éthique. La présence de l'enseignant si souvent évoquée par les élèves 
ne constitue pas encore un objet de réflexion dans le champ de la recherche didactique, il appartient à la phénoménologie de poser des questions sur la puissance perceptive du corps (Merleau-Ponty, 1945, 1961). Cependant, l'enseignant transmet des savoirs dans des postures démonstratives, narratives qui ont une incidence sur le rapport aux savoirs des élèves. Le savoir didactique qui s'institue en classe croise le savoir communiquer, le savoir ajuster en situation, le savoir énonciatif...Une pluralité de savoirs est convoquée par le professionnel et les imbrications de ces savoirs résistent encore à l'analyse. Aussi les questions suivantes ouvrent - elles un espace d'investigation pluridimensionnel :

-Comment l'enseignante prépare t-elle la transition entre la leçon de mathématique et la leçon précédente ? Comment mobilise-t-elle l'attention des élèves ? Quelles sont les ruses, les ficelles qui permettent l'accrochage des élèves ? Quel langage manie-t-elle pour installer un contexte d'apprentissage ? Quelle posture énonciative adopte-t-elle dans la présentation de la tâche ? Comment gère-t-elle les imprévus ?Quels signes d'encouragement, de réprobation adresse-t-elle aux élèves ? Comment met-elle en évidence le savoir didactique ?

Nous tentons, dans l'extrait ici proposé, d'analyser comment l'enseignante sollicite la mémoire didactique des élèves, cela d'un double point de vue, point de vue langagier et point de vue de la corporéité. (Pour une analyse plus longue, Jorro, 2004). Considérons les langages de la mémoire didactique

\section{«Alors vous vous souvenez que hier on a regardé la carte »}

Le plan non - verbal occupe le premier temps. Se situant entre le tableau et la première table, le professeur - stagiaire entre dans un mouvement d'aller-retour pendant lequel elle accorde encore un peu de temps aux élèves qui se préparent à entrer dans la nouvelle séance. Puis, se colle à la première table et s'adresse à la classe. Elle invite les élèves à prendre en compte la carte murale, pour cela elle se recule en faisant pivoter son corps vers l'artefact. Le regard qu'elle porte constitue un signal. Ce geste de désignation de l'artefact doit faire autorité auprès des élèves.

Le geste langagier, volontairement lent, est posé, dans une tonalité basse. Mieux, l'enseignante s'arrête un temps sur le mot « hier » que les élèves repèrent en interagissant sur le mode de l'entente par un «oui ». L'accent didactique est prolongé par l'usage des temps présent / passé. Prenant le soin d'interpeller la classe et de s'impliquer avec les pronoms personnels «vous vous...on », l'instance de rappel est entendue des élèves.

«on a regardé la carte, on a regardé ce qu'il y avait sur cette carte».

Pour fonder la mémoire didactique, l'enseignante reprend la même structure de phrase, sujet verbe - complément, temps passé, référence collective. L'énoncé est particulièrement simple, voire redondant (reprise anaphorique «cette carte, celle que vous avez»). A ce moment précis, il nous semble que l'enseignante déclenche ce que nous avons appelé une entente masquée dans la mesure où elle passe sous silence le champ épistémologique dans lequel elle travaille. L'introduction des élèves dans l'univers des mathématiques n'a pas lieu. Le troisième «seuil » n'est pas franchi. Le geste d'étiquetage qui expliciterait aux élèves l'univers de référence à mobiliser nous semble ici particulièrement absent si bien que la séance sera traversée par une ambivalence : les élèves se situent-ils en mathématiques ou en géographie ? Le langage se développe - t-il à partir d'un univers de références précis ? Il est probable que l'ambiguïté génère des échanges langagiers peu précis associant des mots de sens communs à des concepts scientifiques sans que les élèves ne soient en mesure d'identifier leurs particularités. 


\section{«Alors qui peut me rappeler ce que l'on voit»}

Le marqueur de passage à l'acte " alors » rompt avec la description précédente et signale aux élèves un changement de posture dans le processus de rappel. La question posée sous forme de modalisation entraîne la réaction des élèves. La question posée paraît bancale entre rappel et description, les élèves délaissent la construction intellectuelle attendue pour une énumération. L'enseignante reprend les propositions des élèves, oriente la réflexion sur l'item "numéros». Le registre langagier reste commun jusqu'au moment où un élève propose le concept de distance, que reprendra l'enseignante en répétant la même tournure de phrase "c'est le nombre..., c'est la distance.. » dans un accent didactique qui valide le concept. Le recours au déterminant "la» permet une généralisation qui conduit au processus d'institutionnalisation. Ce travail du langage est complété au plan non verbal. En pointant le doigt sur la carte et en montrant l'espace qui sépare deux villes, l'enseignante matérialise par un geste de représentation le concept de distance.

Au cours de cette situation, l'entente conceptuelle semble toute relative puisque l'enseignante se contente d'assurer le relais (y compris sur le ton didactique) sans favoriser pour autant d'autres prises en charge énonciatives. La distribution de la parole se limite à une interaction avec Yvon, interaction qui servira de tremplin énonciatif pour le reste de la classe. Il est possible de faire un rapprochement avec les travaux de Sirota (1988) sur les réseaux de communication orchestrés par le professeur en classe. Ici, le réseau de communication principal (Enseignant -Yvon) sert de caution à la généralisation. Aussi, le geste d'institutionnalisation qui est créé à partir de la triple répétition «c'est la distance » ne garantit pas la construction du concept par les élèves. L'interaction langagière ici est réduite, l'exploitation langagière de ce concept est quelque peu évacuée. L'ethos de l'enseignant joue ici comme obstacle à la conceptualisation et génère une fausse entente.

LL'enseignante en classe offre une gestuelle porteuse de signaux pour les élèves. Le moindre geste du professeur est perçu, interprété, accepté, refusé et provoque des gestes de l'étude plus ou moins pertinents. Aussi, est-ce du côté de l'épaisseur symbolique de l'agir (Jorro, 2002) que nous orientons notre travail avec l'hypothèse que les gestes professionnels de l'enseignant suscitent des gestes de l'étude. Notre grille d'analyse s'organise autour de la matrice suivante : des gestes langagiers du professeur, des gestes éthiques rendant compte $\mathrm{du}$ format de communication instauré, du format de l'appréciation scolaire, des gestes d'ajustement marquant les régulations mises en œuvres à partir des imprévus de la séance, des gestes de mise en scène du savoir permettent à l'enseignante d'étiqueter la séance, de désigner un objet de savoir, d'institutionnaliser le savoir en question. Mise à l'épreuve de séances de lecture d'album en maternelle, cette matrice de l'agir professoral suppose des variations selon le styles professionnels des enseignants.

\subsection{Définition de la situation en début de cours, le point de vue de l'enseignant stagiaire : une analyse de D. Broussal.}

L'étude suivante va nous permettre d'évoquer la façon dont un enseignant novice perçoit sa propre conduite d'un début de cours. Il s'agit de la même séance d'Ermel évoquée ci-dessus: La carte géographique. Conformément aux propositions d'Ermel ${ }^{10}$, l'intention de l'enseignante est de mener avec des élèves de Ce1 un travail sur la prise d'informations à partir d'un «support qui n'est pas un énoncé ». En l'occurrence, les élèves sont en présence d'une carte géographique de la France, et devront «comparer des trajets en comparant des

\footnotetext{
${ }^{10}$ ERMEL-INRP (1993), Apprentissages numériques - Ce1, Hatier, pp. 74-77.
} 
nombres ». Le fait de travailler en mathématiques sur une carte géographique constitue un brouillage du cloisonnement disciplinaire. Dans la séance d'apprentissage qui leur est proposée, les élèves doivent d'ailleurs résister à un certain nombre d'affordances (Gibson, 1997), d'offres d'utilisation que leur propose la carte du relief affichée au tableau, et dans une moindre mesure le modèle de carte simplifiée dont ils disposent en photocopie. Détaillons quelques unes de ces offres d'utilisation : on peut se servir d'une carte pour situer la France par rapport à des pays limitrophes, activer des connaissances géographiques sur les villes mentionnées, aborder des éléments de relief en décodant une légende (quelques élèves répondront durant la séance à ces diverses affordances).

La définition de la situation spécifique prévue par l'enseignante apparait en ouverture dans une chaîne de reformulations et de schématisations (Grize, 1978), qui s'inscrivent en vingt interactions lors des deux premières minutes du cours. Alors qu'ils sont invités à décrire ce qu'ils voient sur la carte, les élèves parlent tout d'abord de «numéros » et de «pays ». Ces «numéros » et ces «pays » se transforment par guidage de l'enseignante en «nombres » et en « villes », puis enfin en « distances entre les villes ». Ce travail langagier est significatif de la situation, on peut y reconnaître la proposition schématisante, dès lors que l'on a connaissance de l'intentionnalité mathématique. Cette intention n'est cependant pas explicitement formulée lors de la séance.

Parmi les négociations qui vont avoir lieu concernant la définition de la situation, nous nous proposons de rapporter celle qui survient à neuf minutes du début de séance. L'enseignante a demandé aux élèves de trouver un trajet possible entre deux villes : par exemple pour aller de Montpellier à Paris, on peut passer par Lyon ou par Clermont Ferrand. Il s'agit de répondre à l'objectif de prise d'informations, et à celui de choix de trajet qui prépare une étape ultérieure sur la comparaison des distances. La réponse doit être faite en s'aidant de la carte. Or un élève propose un raccourci. D'abord mal compris par l'enseignante, qui pense qu'il s'agit d'une réponse déjà donnée, l'élève insiste :

207 ELEVE : moi je dis que + on passe par Lyon + et on va directement à Orléans + moi je passe pas par Clermont-Ferrand

208 ENSEIGNANTE : dans la réalité on peut aussi

209 ELEVE : mhm

201 ENSEIGNANTE : mais si on suit + si on suit le chemin qui est dessiné sur la carte + ici + t'es obligé de passer par Clermont-Ferrand

L'élève en question utilise donc les savoirs scolaires ou familiaux qu'il a de la géographie de la France pour proposer un trajet qui n'est pas sur la carte. Confrontée à l'enregistrement de ce moment, l'enseignante commente :

ENSEIGNANTE : pour moi euh :: si on a une carte mais ça c'est pf $::+$ on a la carte on regarde la carte donc euh $::+$ on regarde des informations qu'il y a sur la carte

ENQUETTEUR : exactement

ENSEIGNANTE : pour moi + et lui euh ::

ENQUÊTEUR : toi à la question par quel itinéraire on pouvait passer à la limite on pouvait te donner tous les itinéraires?

ENSEIGNANTE : oui

ENQUÊTEUR : même plus longs ?

ENSEIGNANTE : oui

ENQUÊTEUR : mais uniquement ceux qui étaient marqués sur la carte

ENSEIGNANTE : c'est ça

Dans l'auto-confrontation, l'enseignante pointe à plusieurs reprises le décalage sensible entre ce qui lui paraît une évidence de la situation, et la compréhension laborieuse qu'en ont les élèves. Cette prise de conscience a posteriori rejoint, croyons-nous, ce que 
Gérard Sensevy (2001) qualifie de postulation mimétique: «une tendance professorale à considérer en situation que l'élève a compris ce qu'il fallait qu'il comprenne dans les termes où lui (professeur) comprend. » Cette postulation mimétique nous semble être redoublée par la part qui est faite au langage, dans sa participation à la co-définition de la situation. Prenons ainsi les mécanismes de schématisation que nous avons rapidement pointés (de «numéro » et « pays » à « distances entre les villes ») : il ne suffit pas de les produire dans la communauté discursive de la classe, pour que chacun y décèle des attributs définitoires, orientant la séance vers une activité mathématique. Des phénomènes d'indexicalité sont repérables parmi d'autres. Il n'est par certain par exemple que le parcours de «numéro » à «nombre » ou à « distance » soit identiquement interprété par un enseignant en cours de formation, par un didacticien des mathématiques, ou par un élève de Ce1. Mais cette mise en questionnement du fonctionnement du langage dans la définition collective des situations n'apparaît pas dans l'auto-confrontation, pas plus que dans celles que nous avons pu conduire avec d'autres enseignants novices.

Un autre point nous semble interrogeable. Dans son commentaire, l'enseignante perçoit les difficultés qu'ont les élèves à appréhender la situation conformément à son intention. Ils lui adressent de nombreux signaux, témoignant du travail d'enquête auquel ils se livrent. Comme a pu le démontrer Postic (1997), cela n'a rien d'inhabituel : les élèves sont toujours à la recherche des intentionnalités du maître. L'enseignante traite ces interactions des élèves par un ferraillage extrêmement localisé, écartant ce qui doit l'être, procédant par un criblage dont la clé est son intentionnalité. Dans l'auto-confrontation, elle les analyse comme des malentendus, des imprévus qu'elle considère liés à son inexpérience, et au fait qu'elle n'a pas assez anticipé sur diverses difficultés : « là j'ai eu le sentiment d'avoir, d'y avoir pas assez pensé ». De tels événements dans la co-définition de la situation sont donc repérés comme des dysfonctionnements dans la marche de la séance, dysfonctionnements qu'une plus grande expertise permettrait d'éviter, selon l'acteur. Une autre représentation de la situation consisterait à identifier ces événements comme les rouages d'une co-activité opérant en vue de la définition conjointe d'une situation complexe : faire des mathématiques avec un support carte. Ces deux représentations (Vergnaud, 1985) ouvrent, on peut le supposer, sur des modes d'agir différents.

Les auto-confrontations, en nous permettant d'accéder à l'interprétation que font les acteurs des situations d'enseignement, nous renseignent sur les savoirs, concepts, doxas qu'il mobilisent pour élaborer les images opératives (Ochanine, 1981) qui orientent leur action. La confrontation de l'exemple cité, avec le commentaire qu'un enseignant expert peut faire de son début de cours, met en évidence des différences dans la façon dont ces acteurs schématisent les situations, en fonction des buts qu'ils se fixent pour accomplir leur tâche. Ce dialogue entre concepts pragmatiques (Samurçay \& Pastré, 1995) du novice et de l'expert, permet d'identifier dans les débuts de cours un certain nombre de préoccupations expertes qui se rapportent à la construction collective des significations dans la communauté discursive de la classe - qu'est-ce qui s'inscrit et pour qui ? comment les interventions des élèves renseignent sur la qualité de la co-activité ? - opération qui implique fortement les dimensions langagières qu'interroge l'article.

\section{Bibliographie}

ALTET, M. (1994) Comment Interagissent enseignants et élèves dans la classe, Note de synthèse, Revue Française de Pédagogie, $N^{\circ}$ 107, pp. 123-139

BAKHTINE, M. (1979) Esthétique de la création verbale, Paris, Gallimard 
BOIRON, V. (2004) : Conduites et mouvements interprétatifs lors de relecture d'albums et de reprises narratives dialoguées, thèse doctorat non publiée, Paris $\mathrm{V}$.

BRENAS, Y. (2003)Trois seuils pour une installation »épistémologique », Les cahiers du CERFEE, Université P. Valéry

BROUSSEAU, G. (1998) Théorie des situations didactiques. Didatique des mathématiques.

Grenoble, La pensée Sauvage.

BROUSSEAU G. (1986), Fondements et méthodes de la didactique des mathématiques $\boldsymbol{R D M}$ Vol. 7/2, La pensée sauvage.

BUCHETON, D., CHABANNE, J.C., (2002) : Les postures des enseignants débutants , CDROM, Actes du $8^{\circ}$ colloque international de didactique du français,

BUCHETON, D. (2002), Langage, savoirs et subjectivité, Mémoire HDR, non publié,

Université P. Valéry Montpellier

BUCHETON, D., (2003) : Du portfolio au dossier professionnel : éléments de réflexion, TREMA $N^{\circ} 20$, l'Innovation en formation

CHABANNE J.C., BUCHETON, D. (2002), Parler et écrire pour penser, apprendre et se construire. L'écrit et l'oral réflexifs, Paris, PUF

CHALLIES, S., (2002) Analyse des interactions enseignants-stagiaires-conseillers pedagogiques et des connaissances mobilisées et/ou construites lors d'entretiens de conseil pedagogique. Thèse de doctorat non publiée, Montpellier 2

CHEVALLARD Y. (1985) La transposition didactique. Du savoir savant au savoir enseigné,

La Pensée Sauvage.

CHEVALLARD Y. (1992) Concepts fondamentaux de la didactique: Perspectives apportées par une approche anthropologique, RDM Vol 12/1,La Pensée Sauvage.

CHEVALLARD Y. (1999) L'analyse des pratiques enseignantes en théorie anthropologique du didactique, RDM 19/2. La Pensée Sauvage.

CLOT, Y. (1998) La fonction psychologique du travail, Paris PUF

DUVAL R. (1993), Registres de représentation sémiotique et fonctionnement cognitif de la pensée, Annales de Didactique et de Sciences Cognitives, $n^{\circ} 5$. p2 p34 p39 p45

ERMEL (2001) Apprentissages numériques et résolution de problèmes, Hatier

FRANCOIS, F. (2004) Linguistique de la langue et dialogue avec les textes. Un point de vue, La Linguistique, Vol. 39, fasc. 2 /2003

GALIMBERTI, U. (1998) Les raisons du corps. Paris, Grasset.

GIBSON J. (1977), « The theory of affordances », in R. SHAW andJ.BRANSFORD,

Perceiving, acting, and knowing. Toward an ecological psychology, p. 67-82,

Lawrence Erlbaum Associates

GOFFMAN, E.,(1974, traduction française 1991 ) Les cadres de l'expérience, Paris, Minuit

GRIZE J.-B., 1978: Schématisation, représentations et images , in Stratégies discursives,

Presses Universitaires de Lyon, 46 - 52

HANNOUN, H., (1989) Paradoxe sur l'enseigant, ESF

JAUBERT M., REBIERE M. (2002) Parler et débattre pour apprendre : comment caractériser un „oral réflexif“? In CHABANNE J.C., BUCHETON, D. (2002), Parler et écrire pour penser, apprendre et se construire. L'écrit et l'oral réflexifs, Paris, PUF

JAUBERT M., REBIERE M., BERNIE J.P. ed (2003) : Construction des connaissances et langage dans les disciplines d'enseignement. Actes $\mathrm{du}$ colloque pluridisciplinaire international de Bordeaux, 3-5 avril 2003, Bordeaux, Cdrom

JORRO, A. (2004) «Le corps parlant de l'enseignant. Entente, malentendus, négociations ». Symposium «La réflexivité des langages, instruments de travail du professeur et des élèves : points communs et spécificités disciplinaires ». AIRDF. Québec.

JORRO, A. (2002) Professionnaliser le métier d'enseignant. Paris, ESF. 
JORRO, A. (1998) L'inscription des gestes professionnels dans l'action. En Question. Aix-enProvence.

LAVE, J. (1991). Situated learning in communities of practice.In Resnick, L., Levine, J.M., Teadsley, S.D. (eds) Perspectives and socially shared cognition (pp.63-82).Washington : American Psychological Association

LAVE, J., WENGER, E. (1991). Situated learning : legitimate peripheral participation. Cambridge Cambridge University Press

LEMKE J.L., (1997), Cognition, context, learning: a social semiotic perspective in Situated Cognition. Social, semiotic, and psychological perspectives, KIRSHHNER,D.and WHITSON, J. (eds), LEA, London

LUCIANO- BRET, F. (1991) parler à l'école, Ethiques, mobiles et enjeux. Paris, A. Colin MERLEAU -PONTY, M. (1945) Phénoménologie de la perception. Paris, Gallimard.

MERLEAU -PONTY, M. (1960) Signes. Paris, Gallimard.

OCHANINE, D. (1981) L'image opérative. Acte du séminaire Paris 1

PASTRE, P. (2002) L'analyse du travail en didactique professionnelle. Revue française de pédagogie $N^{\circ} 138$

POSTIC, M. (1979) La relation éducative, Paris, PUF

PUJADE - RENAUD, C. (1981) Le corps de l'enseignant dans la classe, Paris, ESF.

SAMURCAY, R. PASTRE, P. (1995). La conceptualisation des situations de travail dans la formation des compétences. Education permanente, 123, 13-31

SIROTA, R. (1988) L'école primaire au quotidien. Paris : PUF.

SENSEVY G. (2001), Modèles de l'action du professeur : nécessités, difficultés , in

Mercier A., Lemoyne G., Rouchier A (dir.), Le génie didactique, Usages et

mésusages des théories de l'enseignement, Bruxelles, De Boeck Université

TOCHON, F.V., (2002) L'analyse de pratiques assistée par vidéo, éditions CRP, université de Sherbrooke

VERGNAUD, G. (1985) Concepts et schèmes dans une théorie opératoire de la représentation. Psychologie française.30, 3-4, pp 245-252 